\title{
Simulation of Longitudinal Multibunch Instabilities in CESR ${ }^{*}$
}

\author{
D. B. Fromowitz, CESR, LNS, Cornell University ${ }^{\dagger}$
}

\begin{abstract}
A tracking simulation predicts beam current thresholds in CESR (Cornell Electron-positron Storage Ring) based on the longitudinal dynamics of multibunch beams in RF cavities. The simulated thresholds are found to have a very strong dependence on the frequencies of the HOM cavity wakefields and are consistent with CESR measurements. Fourier transforms of simulated data are also consistent with observed spectral lines in CESR. After the phase III upgrade to SRF (Superconducting RF) cavities is completed in 1999, a significant increase in the thresholds is predicted.
\end{abstract}

\section{INTRODUCTION}

A new tracking code called "Oscil" [1] was created to study how beam current thresholds are affected by longitudinal motion in CESR. "Oscil" has no transverse degrees of freedom.

Section 2 lists some of the parameters and discusses the physics used in Oscil. Section 3 shows how the simulated thresholds depend on particle and lattice variables. Finally, Section 4 states the theory connecting the growth rates with Fourier spectra of the longitudinal motion.

\section{TRACKING DETAILS}

The Oscil code considers a single beam in a storage ring. Other than RF cavities, there are no explicit optics; the momentum compaction factor $\alpha$ represents all other accelerator elements. Synchrotron radiation effects are included and treated as continuous. Many parameters are specified at run time including, but not limited to, the number of bunches, the bunch current, the number of macroparticles comprising each bunch, and the quantities that define RF cavity modes: $\omega, R / Q$, and $Q_{L}$.

\subsection{Dynamics}

There are four processes that fuel the longitudinal phase space motion of the simulated bunches. The first three processes modulate the bunch energy. First, the bends in the ring cause synchrotron radiation losses. Next, the drive (generator) voltage waveform changes the bunch energy. The third source of energy variations is the cavity wakefields left by previous bunches. This third process is the only way that different bunches (or even macroparticles within a bunch) interact in Oscil. The last phase space ef-

\footnotetext{
* Work supported by the NSF.

† Email: dbf5@cornell.edu
}

fect is the change in the longitudinal displacement between cavities due to a nonzero $\alpha$.

\section{SIMULATION RESULTS}

\subsection{Longitudinal Oscillation Envelopes}

Using a nine-train, two-bunch $(9 \mathrm{x} 2)$ configuration, the envelope of the longitudinal displacement time evolution may be obtained for a variety of conditions. First, if the bunches are pointlike and only fundamental cavity mode wakefields are allowed (i.e. there are no HOM's), then the envelope at $72 \mathrm{~mA}$ total current is that seen in Fig. 1. The beam is highly damped and remains small. (All four cavities have parameters corresponding to copper CESR cavities in this simulation.)

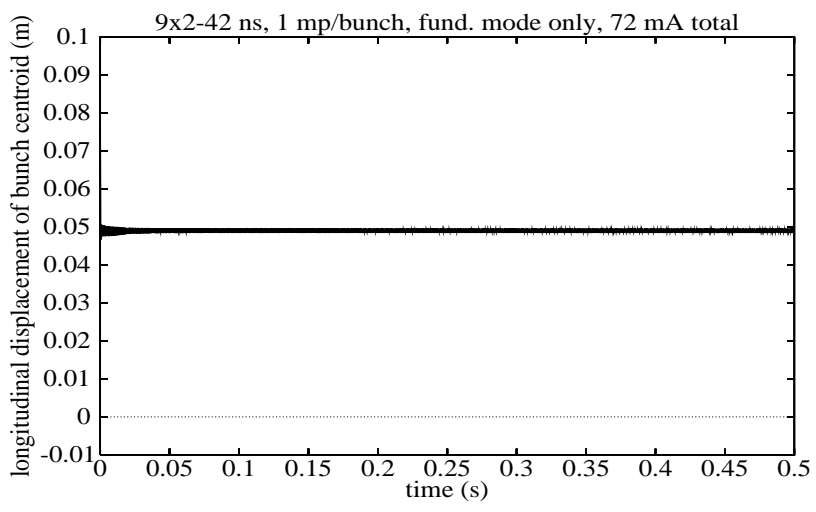

Figure 1: Envelope of longitudinal oscillations of 18 pointlike bunches (with a 42-ns intratrain interbunch spacing) at $72 \mathrm{~mA}$ total current. The only wakefield is that due to the fundamental mode. The interior of the envelope is darkened for clarity.

When the total beam current is increased to $144 \mathrm{~mA}$, the longitudinal displacement undergoes a series of pulses as seen in Fig. 2. (The RF drive wavelength is about 0.60 $\mathrm{m}$.) Although the fundamental cavity and klystron drive frequencies are set such that the machine is Robinson stable, the presence of multibunch modes causes the displacement amplitude to grow occasionally.

As the current is increased even higher, both the period and the amplitude of the oscillations become erratic. At $216 \mathrm{~mA}$ total current, chaos ensues as illustrated in Fig. 3. Remember that this chaos is caused by the fundamental cavity mode alone because there are no HOM's!

Representing a bunch by many macroparticles instead of just one greatly increases the execution time. However, it is necessary to examine multiparticle bunches to see how the behavior changes. When each of the 18 pointlike bunches 


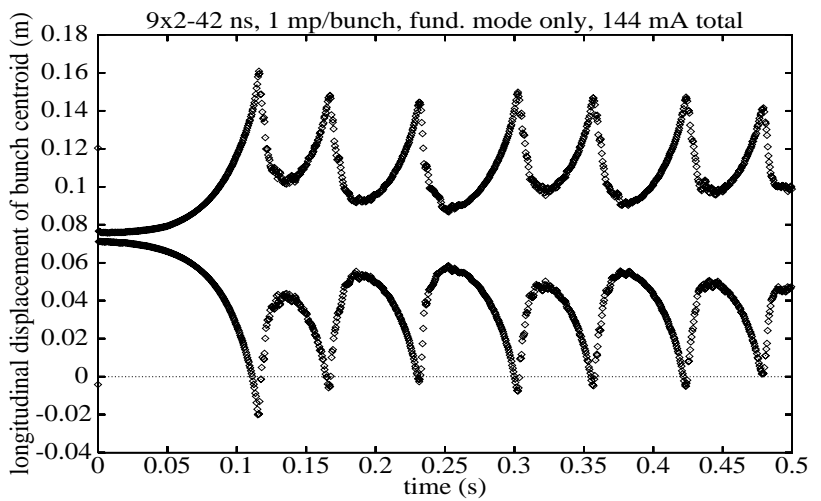

Figure 2: Longitudinal oscillation envelope at $144 \mathrm{~mA}$ total current.

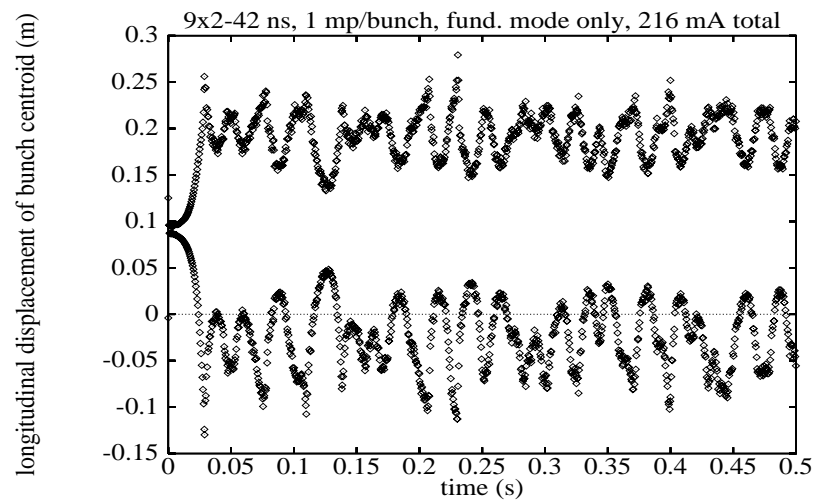

Figure 3: Longitudinal oscillation envelope at $216 \mathrm{~mA}$ total current.

is instead represented by 200 macroparticles, the longitudinal displacement envelope (of the bunch centroid) becomes narrower in several places as a result of Landau damping among the macroparticles.

\subsection{Beam Current Thresholds}

The CESR RF cavities are in the process of being replaced by SRF cavities so that the thresholds may be increased. The main differences between the NRF (normal RF) and SRF cavities are shown in Table 1 . The cavity voltage amplitude $V_{c}$ is one quantity that may vary quite a bit. Table 1 only shows a typical value.

There are two types of thresholds observed with the Oscil code. The lower-current threshold is the current at which the always-damped longitudinal motion (as seen in Fig. 1) turns to a pulsed-amplitude motion (as seen in Fig. 2). This "damping-to-pulses" threshold is plotted in Fig. 4 as NRF cavities are replaced with SRF cavities in a simulation that includes the fundamental and $1399.8 \mathrm{MHz}$ cavity modes. This HOM of about $1400 \mathrm{MHz}$ is used because it has a high $R / Q$ in both NRF and SRF cavities. Parameters of this mode are shown in Table 1.

The most notable feature is that the current thresholds rise as each NRF cavity is replaced with an SRF cavity
Table 1: Differences between normal and superconducting RF cavities in CESR.

\begin{tabular}{|c|c|c|}
\hline Quantity & $\begin{array}{c}\text { NRF }(\mathrm{Cu}) \\
\text { Cavity }\end{array}$ & $\begin{array}{c}\text { SRF }(\mathrm{Nb}) \\
\text { Cavity }\end{array}$ \\
\hline fundamental $Q_{L}$ & 6000 & 200,000 \\
fundamental $R / Q$ & $427 \Omega / \mathrm{m}$ & $145 \Omega / \mathrm{m}$ \\
typical $V_{c}$ & $1.5 \mathrm{MV}$ & $1.9 \mathrm{MV}$ \\
cells per cavity & 5 & 1 \\
$1400 \mathrm{MHz} Q_{L}$ & 1100 & 13.0 \\
$1400 \mathrm{MHz} R / Q$ & $12.6 \Omega / \mathrm{m}$ & $4.7 \Omega / \mathrm{m}$ \\
\hline
\end{tabular}

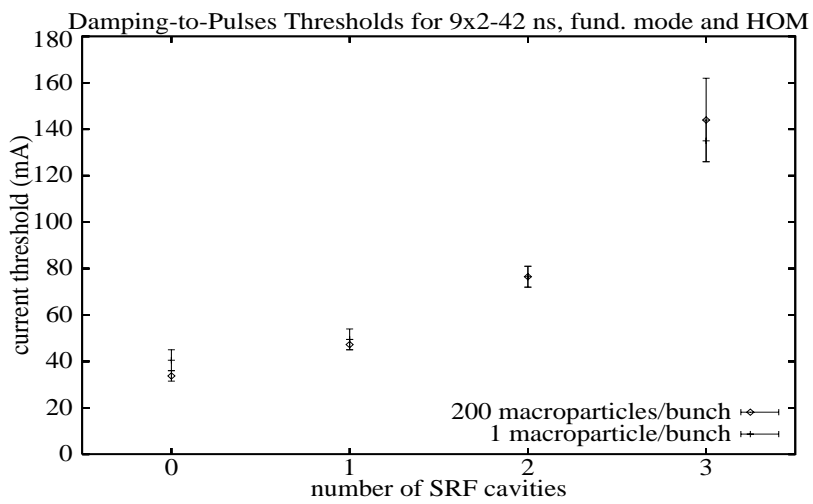

Figure 4: Damping-to-Pulses thresholds for the fundamental mode and a HOM (1399.8 MHz) for pointlike bunches and for bunches of 200 macroparticles.

(with the total number of cavities constant at four). The thresholds are very similar for 1- and 200-macroparticle bunches.

The higher-current threshold is the boundary between the pulsed-amplitude motion (as seen in Fig. 2 or 3) and a higher-current beam where the initial growth rate is so large that the beam immediately diverges. This threshold is coined the "pulses-to-divergence" threshold and is illustrated in Fig. 5.

The pulses-to-divergence threshold also increases with the number of SRF cavities. However, this threshold is markedly higher with multiparticle bunches than with pointlike bunches. The thresholds shown in Fig. 5 for the zero-, one-, and two SRF cavity cases are close to what has been observed in CESR.

\subsection{Cavity Voltage}

The current thresholds recently observed in CESR with one or two SRF cavities were not noticeably higher than when there were no SRF cavities. The reason is that the cavity voltages have often been lowered in CESR as NRF cavities were replaced with SRF cavities. The thresholds introduced in Section 3.2 were based on simulations using the typical $V_{c}$ from Table 1 as the voltages for the cavities. If actual CESR voltages are used in the Oscil simulation, then the simulated thresholds follow the trends of CESR. 


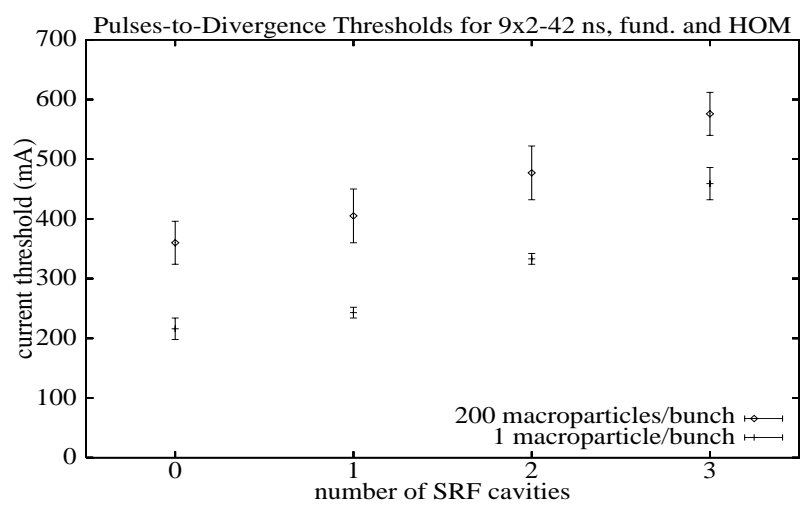

Figure 5: Pulses-to-Divergence thresholds for the fundamental mode and a HOM $(1399.8 \mathrm{MHz})$ for pointlike bunches and for bunches of 200 macroparticles. Compare with the Damping-to-Pulses thresholds of Fig. 4.

\subsection{Effect of HOM's}

The precise values for the thresholds in CESR are not easily duplicated over time. Part of the reason is due to all the different cavity cells present and the differences in temperature of each cell. In reality, there are many different HOM's, and even one particular HOM, such as the 1399.8 $\mathrm{MHz}$ mode, will have a slightly different center frequency in each cell. When the pulses-to-divergence threshold is found for two different HOM conditions, one with all cells having a HOM center frequency of $1399.8 \mathrm{MHz}$, the other condition having $1398.8 \mathrm{MHz}$ for all cells, it is seen that the threshold depends strongly on the HOM frequency, as shown in Fig. 6. Also shown are measured CESR thresholds for zero and one SRF cavity. The 1-SRF-cavity threshold is for $28 \mathrm{~ns}$ spacing and would be higher for $42 \mathrm{~ns}$ and with a higher cavity voltage as explained in Section 3.3.

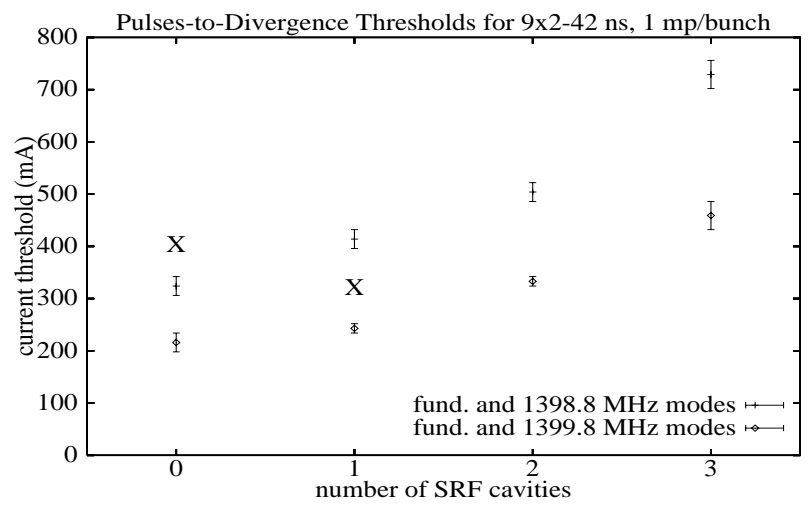

Figure 6: Pulses-to-Divergence thresholds for pointlike bunches with the fundamental cavity mode and one HOM. The upper data set is for a $1398.8 \mathrm{MHz} \mathrm{HOM}$; the lower set for $1399.8 \mathrm{MHz}$. The X's are measured CESR data, the rightmost $\mathrm{X}$ having a $28 \mathrm{~ns}$ interbunch spacing.

This $1 \mathrm{MHz}$ HOM shift corresponds to a $44 \mathrm{~K}$ change in a given cell. Although this is a large temperature fluc- tuation, the actual temperature and shape differences from cell to cell do amount to several $\mathrm{MHz}$ variations, causing a significant threshold variation in time.

\section{GROWTH RATES}

The level of the thresholds are partially explained by the longitudinal growth rates of the beam. Application of a growth rate formula from Chao[2] shows that the initial growth rate is related to the spectral sidebands $(h-1) \omega_{0}+$ $\omega_{s}$ and $(h+1) \omega_{0}-\omega_{s}$ where $h$ is the harmonic number of CESR, $\omega_{0}$ is the revolution frequency, and $\omega_{s}$ is the synchrotron frequency.

If at the first cavity the longitudinal displacement is recorded for every pass of every particle (rather than just for the envelope), then the data may be Fourier transformed to find the sidebands. A spectrum of the first $1 \mathrm{~ms}$ of longitudinal displacement data from the $216 \mathrm{~mA}$ beam represented by Fig. 3 is shown as Fig. 7.

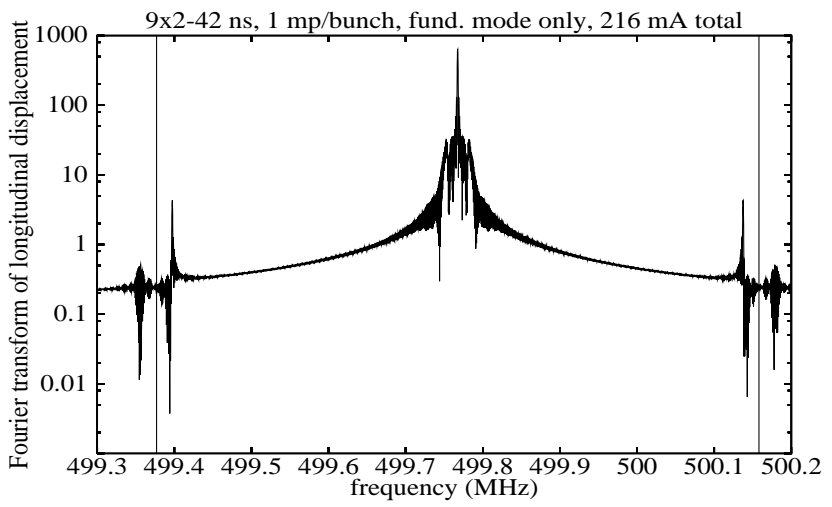

Figure 7: Magnitude of the Fourier transform of the longitudinal displacement of a $216 \mathrm{~mA}$ beam. The vertical lines indicate the $(h \pm 1) \omega_{0}$ revolution harmonics.

The sidebands mentioned are significantly higher than the surrounding features. As a comparison, a spectrum of the $72 \mathrm{~mA}$ beam would show that the indicated sidebands are slightly smaller than the sidebands on the other side of the $(h \pm 1) \omega_{0}$ revolution harmonics. This effect is evidence that the observed instability is associated with the sidebands.

\section{ACKNOWLEDGMENTS}

I appreciate the help and guidance of my research adviser, David Rubin. I thank Zipi Greenwald, Sasha Temnykh, and Sergey Belomestnykh for cavity mode data.

\section{REFERENCES}

[1] D. B. Fromowitz, CBN 98-18 (Laboratory of Nuclear Studies internal paper), Cornell University, 1998.

[2] A. W. Chao, "Coherent Instabilities of a Relatistic Bunched Beam," Physics of High Energy Particle Accelerators 105, 1983. 